The process for obtaining a $T_{\phi}$ containing $\mu$ is now applied to ${A_{2}}^{*}$. Step 1 of this process immediately gives one element of $T_{\phi}$ as element $a_{24}$ of $A$. Step 1 is completed by deleting row 2 , column 4 from $A_{2}{ }^{*}$. This leaves,

$$
A_{21}^{*}=\left(\begin{array}{lll}
1 & 0 & 4 \\
0 & 3 & 0 \\
4 & 0 & 3
\end{array}\right)=\left(\begin{array}{ccc}
a_{11} & 0 & a_{13} \\
0 & a_{32} & 0 \\
a_{41} & 0 & a_{43}
\end{array}\right) .
$$

Element $a_{41}$ of this matrix is now set equal to zero as indicated in Step 1. This new form of $A_{21}^{*}$ satisfies the theorem's hypothesis, so Step 1 is continued by setting $a_{43}$ equal to zero. The matrix $A_{21}^{*}$ now has the following appearance,

$$
A_{21}^{*}=\left(\begin{array}{lll}
1 & 0 & 4 \\
0 & 3 & 0 \\
0 & 0 & 0
\end{array}\right) \text {. }
$$

The hypothesis of the theorem fails for this matrix and so another element of $T_{\phi}$ is $a_{43}$. Deleting row 3 and column 3 from this last matrix leaves,

$$
A_{22}^{*}=\left(\begin{array}{ll}
1 & 0 \\
0 & 3
\end{array}\right)=\left(\begin{array}{cc}
a_{11} & 0 \\
0 & a_{32}
\end{array}\right)
$$

Step 1 is repeated on this matrix, and it is seen that $T_{\phi}$ contains $a_{32}$ and that $A_{23}^{*}=(1)=\left(a_{11}\right)$. From this it follows that the final element of $T_{\phi}$ is $a_{11}$. Therefore, one possible assignment is $T_{\phi}=\left\{a_{11}, a_{24}, a_{32}, a_{43}\right\}$.

As a final remark we note that with obvious simple modifications the algorithm developed here will also solve the analogous problem involving

$$
\mu^{\prime}=\max _{\phi \in \Phi} \min _{a_{i j} \in T_{\phi}} a_{i j} .
$$

Bettis Atomic Power Laboratory

Westinghouse Electric Corporation

Pittsburgh, Pennsylvania

1. P. Hall, “On representatives of subsets," .I. Iond.nn Math. Soc. 10, 1935, p. 26-30.

\title{
Formulas for Integrals of Products of Associated Legendre or Laguerre Functions
}

\section{By James Miller}

1. Introduction. In this paper we derive, using a very simple technique, formulas for the integrals of products of Legendre functions,

$$
\int_{-1}^{1} P_{n_{1}}^{m_{1}}(x) P_{n_{2}}^{m_{2}}(x) \cdots P_{n_{r}}^{m_{r}}(x) d x
$$

Received October 30, 1961. 
where $n_{i}$ and $m_{i}$ are integers and $\sum_{i=1}^{r} m_{i}$ is even, and of Laguerre functions,

$$
\int_{0}^{\infty} e^{-\alpha x} x^{\beta} L_{n_{1}}^{m_{1}}\left(\lambda_{1} x\right) L_{n_{2}}^{m_{2}}\left(\lambda_{2} x\right) \cdots L_{n_{r}}^{m_{r}}\left(\lambda_{r} x\right) d x
$$

where $n_{i}, m_{i}$ and $\beta$ are non-negative integers and $\alpha>0$.

Gaunt [1] has developed a formula for integral (1) when $r=3$. Gillis and Weiss [2] and Gillis and Shimshoni [3] give formulas and describe computational methods for the special case of integral (2) where $r=3, \alpha=3 / 2, \quad \beta=m_{1}=m_{2}=m_{3}=0$, and $\lambda_{1}=\lambda_{2}=\lambda_{3}=1$. Erdélyi [4] gives a general formula for this integral. Neither the general Erdélyi formula for integral (2) nor the special Gaunt formula for integral (1), however, are particularly well suited for programming for an electronic computer. In view of the importance of special cases of these integrals (in addition to the case treated in references [2] and [3]) in theoretical physics $[1,5,6]$, it is desirable to have easily programmed expressions. Moreover, the simplicity of the present approach in developing general formulas for these integrals may itself be of some interest.

2. Formula for the Integral of the Product of Associated Legendre Functions of the First Kind. Taking as the definition of the associated Legendre function

$$
P_{n}^{m}(x) \equiv \frac{\left(1-x^{2}\right)^{m / 2}}{2^{n} n !} \frac{d^{m+n}}{d x^{m+n}}\left(x^{2}-1\right)^{n}
$$

one can obtain the expression

$$
P_{n}^{m}(x)=\left(1-x^{2}\right)^{m / 2} \sum_{i=0}^{I(m, n)} c_{i}(m, n) x^{n-m-2 i}
$$

where $m$ and $n$ are integers, $n \geqq 0$, and $-n \leqq m \leqq n$;

$$
c_{i}(m, n)=\frac{(-1)^{i}[2(n-i)] !}{2^{n}(n-m-2 i) !(n-i) ! i !},
$$

and $I(m, n)=$ integer part of $\frac{1}{2}(n-m)$. Let

$$
P_{n_{1} n_{2} \cdots n_{r}}^{m_{1} m_{2} \cdots m_{r}}=\int_{-1}^{1} P_{n_{1}}^{m_{1}}(x) P_{n_{2}}^{m_{2}}(x) \cdots P_{n_{r}}^{m_{r}}(x) d x
$$

and substitute in (3):

$$
\begin{aligned}
P_{n_{1} n_{2} \cdots n_{r}}^{m_{1} m_{2} \cdots m_{r}} & =\int_{-1}^{1}\left(1-x^{2}\right)^{M / 2} \sum_{i=0}^{I\left(n_{1}, m_{1}\right)} \sum_{j=0}^{I\left(n_{2}, m_{2}\right)} \\
& \cdots \sum_{k=0}^{I\left(n_{r}, m_{r}\right)} c_{i}\left(n_{1}, m_{1}\right) c_{j}\left(n_{2}, m_{2}\right) \cdots c_{k}\left(n_{r}, m_{r}\right) \cdot x^{N-M-2(i+j+\cdots+k)} d x
\end{aligned}
$$

Here $M=\sum_{i=1}^{r} m_{i}, \quad N=\sum_{i=1}^{r} n_{i}$.

We wish to expand $\left(1-x^{2}\right)^{M / 2}$ binomially and multiply the resulting polynomial by the other polynomial in the integrand of (4). If the expansion of $\left(1-x^{2}\right)^{M / 2}$ is to terminate, $M$ must be even and non-negative. (For many physics problems of interest, $M$ is even.) If $M$ is negative, we may use the identity

$$
P_{n}^{-m}(x) \equiv(-1)^{|m|} \frac{(n-m) !}{(n+m) !} P_{n}^{+m}(x)
$$


as many times as necessary to render $M$ non-negative. Then

$$
\begin{aligned}
P_{n_{1} n_{2} \cdots n_{r}}^{m_{1} m_{2} \cdots m_{r}}= & \int_{-1}^{1} \sum_{q=0}^{M / 2} \sum_{i=0}^{I\left(n_{1}, m_{1}\right)} \sum_{j=0}^{I\left(n_{2}, m_{2}\right)} \cdots \sum_{k=0}^{I\left(n_{r}, m_{r}\right)}(-1)^{q} \\
& \cdot\left(\begin{array}{c}
M / 2 \\
q
\end{array}\right) c_{i}\left(n_{1}, m_{1}\right) c_{j}\left(n_{2}, m_{2}\right) \cdots c_{k}\left(n_{r}, m_{r}\right) \cdot x^{N-M-2(i+j+\cdots+k-q)} d x .
\end{aligned}
$$

Integrating gives

$$
\begin{aligned}
P_{n_{1} n_{2} \cdots n_{r}}^{m_{1} m_{2} \cdots m_{r}}=\sum_{q=0}^{N / 2} \sum_{i=0}^{I\left(n_{1}, m_{1}\right)} \sum_{j=0}^{I\left(n_{2,}, m_{2}\right)} \cdots \sum_{k=0}^{I\left(n_{r}, m_{r}\right)}(-1)^{q} \\
\cdot\left(\begin{array}{c}
M / 2 \\
q
\end{array}\right) c_{i}\left(n_{1}, m_{1}\right) c_{j}\left(n_{2}, m_{2}\right) \cdots c_{k}\left(n_{r}, m_{r}\right) \\
\cdot[N-M-2(i+j+\cdots+k-q)+1]^{-1}\left\{1-(-1)^{[N-M-2(i+j+\cdots+k-q)+1]}\right\} .
\end{aligned}
$$

This expression vanishes unless the exponent of $(-1)$ is odd. Since

$$
-M-2(i+j+\cdots+k-q)+1
$$

is always odd, $N$ must be even.

Define:

$$
\delta(\text { even, } N)=\left\{\begin{array}{llll}
1 & \text { if } & N & \text { is even } \\
0 & \text { if } & N & \text { is odd }
\end{array}\right.
$$

Then

$$
\begin{aligned}
& P_{n_{1} n_{2} \cdots n_{r}}^{m_{1} m_{2} \cdots m_{r}}=2 \delta(\text { even, } N) \sum_{q=0}^{M / 2} \sum_{i=0}^{I\left(n_{1}, m_{1}\right)} \sum_{j=0}^{I\left(n_{2}, m_{2}\right)} \cdots \sum_{k=0}^{I\left(n_{r}, m_{r}\right)}(-1)^{q} \\
& \cdot\left(\begin{array}{c}
M / 2 \\
q
\end{array}\right) c_{i}\left(n_{1}, m_{1}\right) c_{j}\left(n_{2}, m_{2}\right) \\
& \cdots c_{k}\left(n_{r}, m_{r}\right)[N-M-2(i+j+\cdots+k-q)+1]^{-1} .
\end{aligned}
$$

Programming this expression for a computer may be facilitated by using the relations

$$
\begin{aligned}
c_{i+1}(n, m) & =-\frac{(n-m-2 i)(n-m-2 i-1)}{2[2(n-i)-1](i+1)} c_{i}(n, m) \\
c_{i}(n+1, m) & =\frac{2(n-i)+1}{n-m-2 i+1} c_{i}(n, m) \\
c_{i}(n, m+1) & =(n-m-2 i) c_{i}(n, m) .
\end{aligned}
$$

3. Formula for the Integral of the Product of Associated Laguerre Polynomials with Arbitrary Weight Function. Taking as the definition of the associated Laguerre polynomial*

$$
L_{n}{ }^{m}(x) \equiv \frac{d^{m}}{d x^{m}}\left[e^{x} \frac{d^{n}}{d x^{n}}\left(x^{n} e^{-x}\right)\right]
$$

* This is the definition usually given in physics books. In mathematical works, the right hand side is multiplied by $\frac{1}{n !}$. 
one may obtain the expression

$$
L_{n}{ }^{m}(x)=\sum_{i=m}^{n} b_{i}(n, m) x^{i-m}
$$

where $0 \leqq m \leqq n$ and $b_{i}(n, m)=(-1)^{i} \frac{(n !)^{2}}{(n-i) !(i-m) ! i !}$ where $m \leqq i \leqq n$. Let

$$
L_{n_{1} n_{2} \cdots n_{r}}^{m_{1} m_{2} \cdots m_{r}}=\int_{0}^{\infty} e^{-\alpha x} x^{\beta} L_{n_{1}}^{m_{1}}\left(\lambda_{1} x\right) L_{n_{2}}^{m_{2}}\left(\lambda_{2} x\right) \cdots L_{n_{r}}^{m_{r}}\left(\lambda_{r} x\right) d x
$$

where $\alpha>0$ and $\beta$ is a non-negative integer. Substituting in expression (5) and integrating

$$
\begin{aligned}
L_{n_{1} n_{2} \cdots n_{r}}^{m_{1} m_{2} \cdots m_{r}}=\sum_{i=m_{1}}^{n_{1}} \sum_{j=m_{2}}^{n_{2}} \cdots \sum_{k=m_{r}}^{n_{r}} \lambda_{1}^{i-m_{1}} b_{i}\left(n_{1}, m_{1}\right) \cdot \lambda_{2}^{j-m_{2}} b_{j}\left(n_{2}, m_{2}\right) \\
\cdots \lambda_{r}^{k-m_{r}} b_{k}\left(n_{r}, m_{r}\right) \frac{(i+j+\cdots+k+\beta-M) !}{\alpha^{(i+j+\cdots+k+\beta-M+1)}}
\end{aligned}
$$

where $M=\sum_{i=1}^{r} m_{i}$. Programming this expression for a computer may be facilitated by using the relations

$$
\begin{aligned}
b_{i+1}(n, m) & =-\frac{(n-i)}{(i+1)(i-m+1)} \quad b_{i}(n, m) \\
b_{i}(n+1, m) & =\frac{(n+1)^{2}}{(n-i+1)} \quad b_{i}(n, m) \\
b_{i}(n, m+1) & =(i-m) \quad b_{i}(n, m) .
\end{aligned}
$$

4. Acknowledgements. I wish to thank Professor F. A. Matsen of the University of Texas for his support during the preparation of a major part of this paper. Thanks go also to Dr. J. C. Browne, Dr. I. Shavitt, Dr. R. P. Hurst, and Mr. M. Tainiter for helpful comments.

International Business Machines Corporation

Thomas J. Watson Research Center

Yorktown Heights, New York

1. J. A. Gaunt, Philos. Trans. Roy. Soc. London, v. A228, 1929, p. 194. p. 60 .

2. J. Gillis \& G. Weiss, "Products of Laguerre polynomials," Math. Comp., v. 14, 1960,

3. J. Gillis \& M. Shimshoni, "Triple product integrals of Laguerre functions," Math. Comp., v. 16, 1962, p. 50.

4. A. ERDElyi, Math. Z., v. 40, 1936, p. 693-702.

5. E. U. Condon \& G. H. Shortley, The Theory of Atomic Spectra, Cambridge University Press, Cambridge, 1953, p. 175.

6. J. C. Browne \& J. Mrller, J. Chem. Phys., v. 36, no. 9, 1962, p. 2324-5. 